\title{
Excretion of biogenic selenium nanoparticles depends on outer membrane integrity in Escherichia coli
}

\author{
ANNA OCHI ${ }^{1}$, RYUTA TOBE ${ }^{1}$, YOSUKE TOYOTAKE ${ }^{1}$, \\ YUKIKO IZU $^{1}$, TOMOYA IMAI ${ }^{2}$ AND HISAAKI MIHARA ${ }^{1}$ \\ ${ }^{1}$ Ritsumeikan University \\ ${ }^{2}$ Kyoto University \\ Presenting Author: annaoti009@yahoo.co.jp
}

A variety of bacteria can reduce toxic selenite $\left(\mathrm{SeO}_{3}{ }^{2-}\right)$ into less-toxic elemental selenium $\left(\mathrm{Se}^{0}\right)$ that is extracellularly accumulated as red selenium nanoparticles (SeNPs). Selenite reduction is thought to occur intracellularly. However, the mechanisms underlying the formation and the subsequent excretion of SeNPs are largely unknown. Because E. coli is a gram-negative bacterium, which has outer membrane (OM), OM is believed to be involved in the mechanism of excretion of SeNPs. E. coli is known to produce outer membrane vesicles (OMVs), which are spherical buds with sizes of 20-300 $\mathrm{nm}$ in diameter of the outer membrane filled with periplasmic content. It has been reported that an E. coli mutant lacking one of the major cell envelope proteins, OmpC, TolA, and NlpI, which are important for membrane integrity, exhibit increased OMVs synthesis. In this study, we investigated the association between OMVs and extracellular SeNPs formation.

E. coli BW25113 (wild type, WT) was cultured in a medium containing selenite, and extracellular SeNPs were purified from the culture supernatant by centrifugation. Atomic force microscopy analysis showed that SeNPs were spherical in shape with diameters between 40 and $100 \mathrm{~nm}$. SDS-PAGE analysis of the isolated SeNPs revealed that proteins including the major OM proteins, OmpC and OmpA, are bound to SeNPs. When OMVs-overproducing mutant strains, $\Delta o m p C, \Delta t o l A$, and $\Delta n l p I$, were cultivated in the presence of selenite, they exhibited increased selenite reducing ability as compared with WT. Interestingly, we found that $\triangle o m p C$ and $\triangle t o l A$ were unable to produce extracellular SeNPs; instead, they appeared to accumulate SeNPs inside the cells. Moreover, SeNPs purified from the lysate of $\triangle o m p C$ showed relatively larger sizes $(>300$ $\mathrm{nm}$ in diameter) of nanoparticles as compared with those extracellularly formed by WT. These results suggest that the integrity of OM is important not only for the synthesis of normalsized SeNPs but also for cellular excretion of SeNPs. 